\title{
Measurement of Volumetric Flow
}

\author{
Oliver D. Kripfgans, PhD, Jonathan M. Rubin, MD, PhD, \\ Anne L. Hall, PhD, Michael B. Gordon, MD, PhD, \\ J. Brian Fowlkes, PhD
}

\begin{abstract}
Objective. The purpose of this study was to evaluate a 3-dimensional (3D) sonographic method for the measurement of volumetric flow under conditions of known flow rates and Doppler angles. Methods. A GE/Kretz Voluson 730 system (GE Healthcare, Milwaukee, WI) and RAB2-5 probe were used to acquire 3D Doppler measurements in a custom flow phantom. Blood-mimicking fluid circulated by a computer-controlled pump provided a range of flow velocities $(2-15 \mathrm{~mL} / \mathrm{s})$. A 6 -axis positioning system maneuvered the ultrasound probe through a range of angles $\left(40^{\circ}-70^{\circ}\right.$ and $\left.110^{\circ}-140^{\circ}\right)$ with respect to the tube (orthogonal to the tube being $90^{\circ}$ ). Volume data sets were obtained spanning $29^{\circ}$ lateral and $20^{\circ}$ elevational angles encompassing the flow tube in a scanning time of less than 10 seconds. Power Doppler data were used to correct for partial volume effects. Results. Using a single angle $\left(110^{\circ}\right)$ with respect to the flow tube, measured and actual volume flow rates were within the $95 \%$ confidence interval over the full range of flow rates. At flow rates of 5 and $10 \mathrm{~mL} / \mathrm{s}$, the measured volume flow rates were all within $\pm 15 \%$ of actual values for the range of angles tested and also stayed within the $95 \%$ confidence interval. Conclusions. Direct comparisons of volume flow rates estimated with 3D sonography and known flow rates showed that the method has good accuracy. Subsequent comparisons under pulsatile and in vivo conditions will be needed to verify this performance for clinical applications. Key words: color flow; Doppler sonography; flux; vector Doppler sonography; volume flow.
\end{abstract}

\section{Abbreviations}

$\mathrm{CF}$, color flow; ROI, region of interest; SIVV, surface integration of velocity vectors; 3D, 3-dimensional; VOI, volume of interest
Received April 27, 2006, from the Department of Radiology, University of Michigan, Ann Arbor, Michigan USA (O.D.K., J.M.R., M.B.G., J.B.F.); and GE Healthcare, Milwaukee, Wisconsin USA (A.L.H.). Revision requested June 8 , 2006. Revised manuscript accepted for publication June 15, 2006.

This work was supported in part by National Institutes of Health grant R01 HL67921 and by GE Healthcare.

Address correspondence to Oliver Kripfgans, PhD, Department of Radiology, University of Michigan, Kresge III, R3322, Ann Arbor, MI 48109-0553 USA.

E-mail: oliver.kripfgans@umich.edu

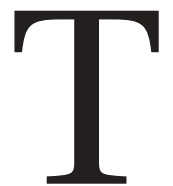

he standard method for estimating volume flow using sonography consists of multiplying the mean spatial velocity by the luminal crosssectional area. ${ }^{1}$ However, it is well known that this technique has many problems. These include inherent variability of vessel geometry, assumptions about flow profile, 1-dimensional sampling, and others as well. ${ }^{2}$ Burns $^{2}$ specifically addressed difficulties of Doppler assessment of physiologic quantities due to the inherent variability of vessel geometry and flow profile. The mean spatial velocity also has limitations. First, it requires knowledge about the Doppler angle, and second, there are errors associated with the spatial resolution of the narrow-band/long-tone burst Doppler pulse used. In addition, the method assumes a circular cross section. Other schemes include the acquisition of the Doppler power, in which 2 Doppler acquisitions are obtained, 1 with a narrow beam width (completely within the interior of the vessel) and 1 large-diameter beam covering the entire lumen. ${ }^{3}$ Assuming that the sampled vessel's interior volume is represented by the amount of signal power 
as signal power per unit of blood volume, one can estimate the vessel cross section without assuming any specific shape. In this way, the vessel cross section need not be known. However, this technique might encounter problems as deeper vessels are interrogated. ${ }^{2}$ Also, red blood cell rouleaux will invalidate the assumption that the narrow beam signal will represent a typical blood volume. ${ }^{4}$ Speckle tracking has been proposed to measure flow based on the coherent backscatter of red blood cells. ${ }^{5}$ Positive aspects of this method include the short acoustic pulse, which leads to greater spatial resolution. However, this method still assumes a nearly symmetric flow distribution and a circular vascular cross section. Yet, diagnostic examinations of volume flow would be beneficial in the areas of cardiac, carotid, renal, and splanchnic circulations of the adult and fetal placental as well as aortic and cardiac circulations. ${ }^{2}$

Physiologic quantities such as blood volume flow are desired and find themselves on the verge of clinical use. This will be possible through the ongoing development of 3-dimensional (3D) sonographic imaging. Recent introductions of true 2-dimensional transducer arrays as well as the currently widely available mechanically scanned arrays enable the acquisition of true, angle-independent blood volume flow. We and others have described the method of surface integration of velocity vectors (SIVV) to estimate volume flow. ${ }^{6-9}$ This method will be described here along with measurements showing the quality of volume flow estimation and angle independence. ${ }^{4,10}$

\section{Materials and Methods}

\section{Experimental Setup}

A GE/Kretz Voluson 730 sonography system for 3-dimensional static/4-dimensional real-time imaging (GE Healthcare, Milwaukee, WI) was used to acquire flow in a Doppler phantom. The sonographic scanner was equipped with standard control software, which allowed the user to store 3D duplex mode data as well as associated scanner settings. Scans were performed with a 2to 5-MHz wideband convex volume array (RAB25), intended for abdominal and obstetric/gynecologic examinations. A 6-axis computer-controlled stepper motor positioning setup held the transducer array to allow tube alignment and angle control between the array and a flow tube. ${ }^{11}$
Blood-mimicking fluid was purchased from Shelley Medical Imaging Technologies (London, Ontario, Canada) and is advertised as providing the complex properties of blood. It is compatible with sonographic, magnetic resonance, and radiographic imaging techniques.

A CompuFlow 1000 System (Shelley Medical Imaging Technologies) was used to circulate the blood-mimicking fluid. This system uses a positive-displacement pump driven by a microstepping motor, under the control of an embedded microprocessor, resulting in accurate, steady flow (here $2-15 \mathrm{~mL} / \mathrm{s}$ ) and highly reproducible pulsatile waveforms. However, only constant flow was used in this study. Thin-walled natural rubber tubing $(9.5 \mathrm{~mm}$, 0.375 -inch diameter and $380 \mu \mathrm{m}, 0.015$-inch wall thickness; Kent Elastomer Products Inc, Kent, $\mathrm{OH}$ ) was embedded in a tissue-mimicking phantom (custom phantom from CIRS, Norfolk, VA; $\alpha=0.3 \mathrm{~dB} \cdot \mathrm{MHz}^{-1} \cdot \mathrm{cm}^{-1}$ ). The described setup allowed the user to tilt the transducer array with respect to the flow tube and achieve a range of Doppler angles. This range is in addition to what is inherently possible because of the natural curvature of the transducer face used.

Figure 1 illustrates how the left and right edges of the region of interest (ROI) are on distinctively different angles to the tubing. Close to the right edge of the flow tube, the Doppler angle is nearly perpendicular to the flow so the color flow (CF) tapers off as the registered flow transitions into the wall filter. In addition, the measured velocities increase toward the left side of the CF ROI as the Doppler angle approaches $0^{\circ}$.

\section{Data Acquisition and Analysis}

Figure 1, left, shows conventional duplex mode $\mathrm{B}$-mode and overlaid CF imaging. Conventional CF ROI positioning in the duplex image (Figure 1 , left) is followed by the out-of-plane/elevational volume of interest (VOI) alignment with the flow tube. Shown in Figure 1, right, are the intersecting orthogonal image planes: axial-lateral, axial-elevational, and lateral-elevational (A-C, respectively). Typically a $30-$ to $200-\mathrm{mL}$ scan volume resulted from the VOI selection. The 3D CF VOI in Figure 1 was set to $29^{\circ}$ lateral and $20^{\circ}$ elevational angles, which encompassed the entire flow tube, including additional nonflowing material in the 3D sweep, thus reducing the scan time ( $<10$ seconds). 


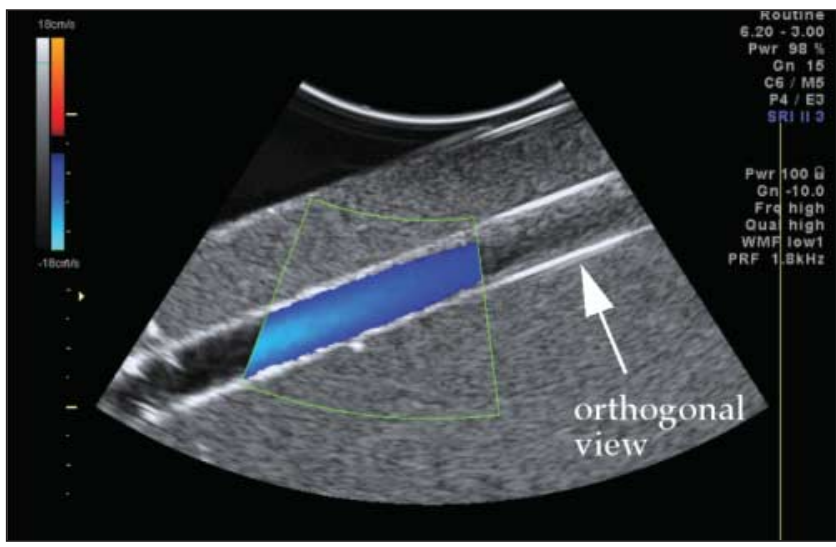

Figure 1. Left, The conventional duplex mode with B-mode and overlaid CF is shown. The measured flow profile narrows as the beam-to-tube angle approaches $90^{\circ}$ (arrow) and the relative Doppler velocities fall into the wall filter. Note the increased (static) echogenicity inside the tube toward the right. It is a result of transducer array-to-tube alignment as well as a larger elevational slice thickness of the acoustic beam at this depth. Right, The 3D CF acquisition mode of the Voluson 730 system was set to a CF VOI of $29^{\circ}$ lateral and $20^{\circ}$ elevational angles. Intersecting orthogonal image planes (A, axial-lateral; B, axial-elevational; and C, lateral-elevational) are shown.

After data were exported to an off-line computer, MATLAB (The MathWorks Inc, Natick, MA) was used to define a cross-sectional surface through the flow tube. Figure 2 shows axiallateral and axial-elevational views of the data set. For display purposes, the ROI was rotated to be symmetric to the vertical. The asterisk lines depict 2 cross sections of this surface, whereas Figure 3 shows the 3D surface itself (not to scale).

Surface integration of velocity vectors is based on Gauss' theorem, which relates the divergence of the quantity $v$ in an enclosed volume $V$ to the flux through the surface $S$ covering $V$. In other words, a surface integral of $v$ over the enclosing boundary $S$ will yield the volume flow $Q$ :

$$
Q=\int_{S} \vec{v} \cdot \overrightarrow{d A}
$$

The easiest way to implement SIVV is to choose a surface that is locally perpendicular to each Doppler beam. For this case, the right side dot product in Equation 1 will be replaced by a regular multiplication of the Doppler velocity and the size of the surface element. In other words, this surface has a constant depth geometry with respect to the Doppler beams. However, SIVV is not limited to this choice. It can be shown that this surface can be tilted by a known angle when the resulting velocity values are compensated for this tilt. Note that this is not the classic Doppler angle correction and requires no a priori knowl- edge of the flow direction. The most general scanning geometry is the surface of a torus because the center of rotation for the axial-lateral and axial-elevational beams can differ (as shown in Figure 3). Volume flow is computed by integrating all Doppler acquisitions on the defined surface such that

$$
Q=\sum_{i \in S} v_{i} \cdot a_{i}
$$

Here, $Q$ is the total flux through the surface $S$, which equals volume flow; $v_{i}$ is the local Doppler velocity; $a_{i}$ is the associated cross-sectional area on $S$ for this voxel, and - is the dot product between the local velocity vector and the local surface normal. In our case, the detected velocity is parallel to the surface normal.

As pointed out already, the Doppler angle for a sector scan format varies with each beam. Figure 4 illustrates how a symmetric flow profile will be mapped onto the measured Doppler shift profile. For the depicted type of tube-to-transducer orientation, the left-/right-most Doppler beam has the smallest/largest Doppler angle, respectively. This causes the velocity maximum to shift toward the left. However, warping of velocity values does not affect the measured SIVV value. Inherent compensation of this warping is due to nonuniform distribution of surface elements over the tube cross section. In Figure 4, one can see that the Doppler beam voxel distribution across the tube is denser toward the right-side 


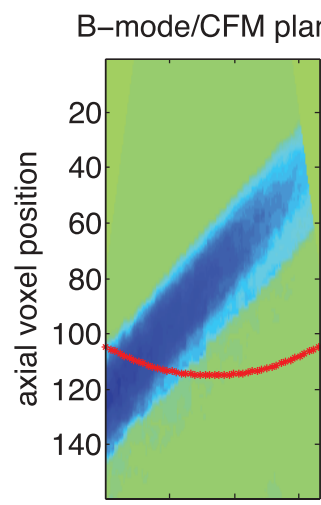

50100150 lateral voxel position

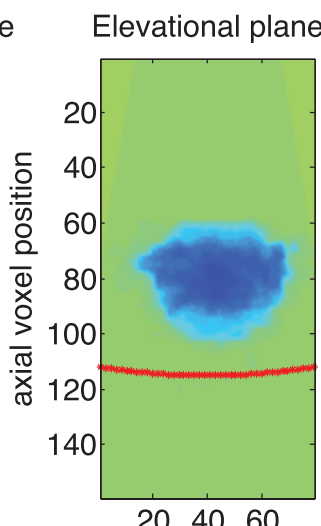

$2040 \quad 60$
Figure 2. Axial-lateral and axial-elevational cross sections through a CF data set with indicated cross sections through a chosen 3D surface for SIVV. The displayed surface is of a constant depth geometry and shown in 3D in Figure 3.

Doppler beams. Hence, it can be understood that this effect might cause the SIVV value to remain constant.

\section{Power Weighting}

The local Doppler power in our measurements was largest inside the vessel and smallest outside because of the effect of partial volume averaging and the Doppler wall filter effect, which gives the pixels inside the vessel a higher power. All Doppler data were wall filter processed internally in the

Figure 3. To be very general, a constant depth geometry is the surface of a torus because the center of rotation for the axial-lateral and axial-elevational beams can differ.

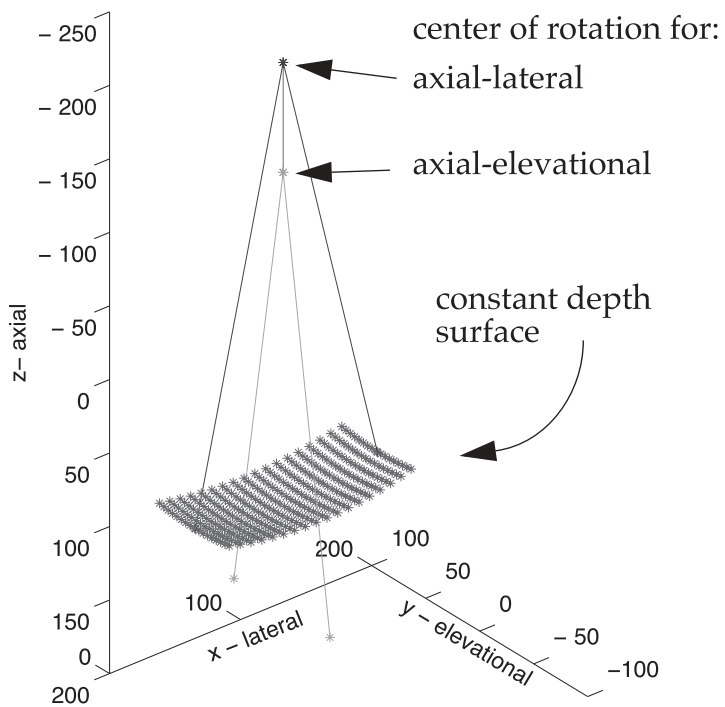

scanner by the smallest setting possible on the scanner. We chose this setting to minimize filtering for the selected Doppler frequency range. Unfortunately, more specific data on the filter in terms of frequency or velocity were not displayed by the Voluson 730 system used. Then Doppler power values from the surface for integration was used to compute a Doppler power histogram shown in Figure 5A. Two power values were computed for subsequent processing. The maximum of the histogram, indicated by $p_{M}$, was computed first. Then a power threshold, $p_{T}$, was selected as $90 \%$ of the Doppler power of $p_{M}$.

Surface integration of velocity vectors was weighted on the basis of the Doppler power in the respective voxels. An automatic velocitymasking algorithm was generated, which uses the above Doppler power value $p_{T}$ to weight the velocity values of the integration surface. Therefore, a modified SIVV method was used to compute our results (Equation 3) as

$$
Q=\sum_{i \in S}\left(v_{i} \cdot a_{i}\right) p_{i}
$$

Figure 4. Tube flow profiles are projected onto the individual Doppler firings. A curvilinear array will have a range of Doppler angles because the individual firings are along various angles within the sector format. This leads to a warp in the perceived flow profile symmetry. The asterisk line represents the chosen surface over which the Doppler velocities are integrated. At each Doppler firing beam, the surface is normal to that beam.

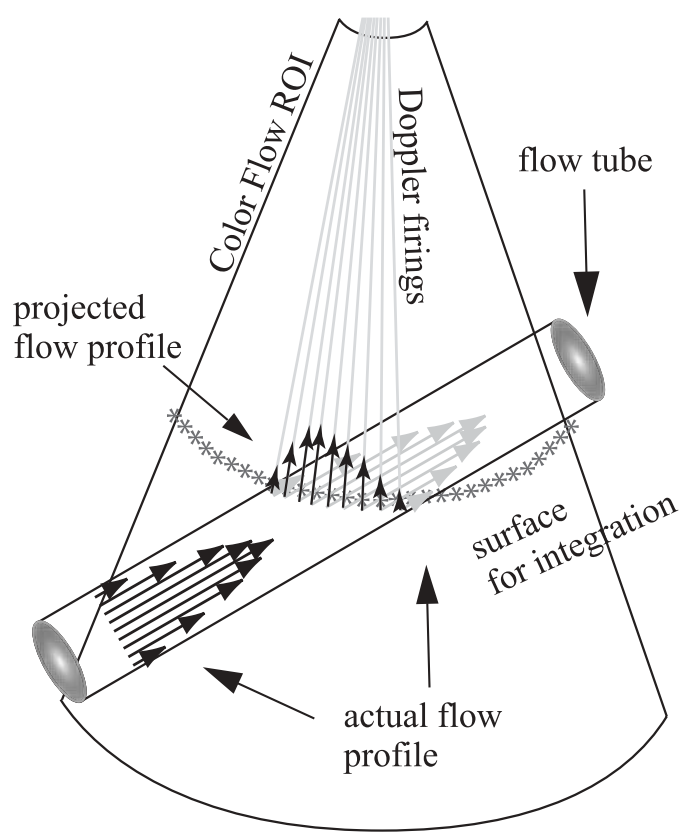


Here, $p_{i}$ is a scaling factor based on the local Doppler power. Power weighting factors $p_{i}$ were set to 1 for all Doppler powers larger then $p_{T}$. Values between 0 and $p_{T}$ were scaled between 0 and 1 on the basis of their Doppler power value. The justification for the selection/scaling process is that voxels near the wall, which partially contain flow and soft tissue, will show lowerthan-maximum Doppler power. Fractional power is therefore weighted by fractional scaling factors. So far, the threshold $p_{T}$ had been set empirically. However, it was set constant for all measurements, and it will be shown that the $p_{T}$ contour fills the lumen. User-selected Doppler gain was adjusted as needed to compensate for signal reduction due to large angles between flow and Doppler beams.

\section{Statistical Analysis}

The data presented were statistically analyzed to determine their confidence interval. Measure- ments on varying volume flow were fitted to a linear function, $Y=a \cdot X+b$, and measurements on varying Doppler angles were first normalized by the pump setting to yield a value close to unity (indicated by the normalized unit $[\mathrm{mL} / \mathrm{s}$ per $\mathrm{mL} / \mathrm{s}]$ ) and then fitted to a linear function. For each regression parameter $a$ and $b$, the fitted value and the SEM were determined.

\section{Results}

A toroidal surface with Doppler power, $\mathrm{CF}$, and B-mode can be seen in Figure 5, B-D. The Doppler power remains constant inside the vessel (Figure 5B), except for loss along the wall due to partial voluming and attenuation effects. The $p_{T}$ contour encloses only a fraction of the total power and CF region (Figure 5, B and C). All velocities inside this contour were weighted by 1 , whereas all velocities outside this contour were weighted with values between 0 and 1 , depend-

Figure 5. Surface integration of velocity vectors is weighted by the associated Doppler power. On the basis of the Doppler power histogram $(\mathbf{A})$, velocities are scaled between 0 and 1 to compensate for a partial voluming effect, in which voxels are not fully inside or fully outside the vessel/tube (below the power value $p_{T}=0.9 \cdot p_{M}$; here, $p_{T}=202$ ). Doppler power remains constant inside the vessel, and power levels at $p_{T}$ are indicated by the contour line in the power image (B). The same contour is also given on the corresponding CF and B-mode images (B and $\mathbf{C}$ ). For both power and $C F$, the $p_{T}$ contour line encloses only a fraction of the shown power or CF. However, superpositioning of the contour and the B-mode image (D) shows that the $p_{T}$ criterion approximately encompasses the entire lumen. The misregistration between power/CF mode and B-mode is illustrated by the red (original) and white (centered on the lumen) contour lines.

A

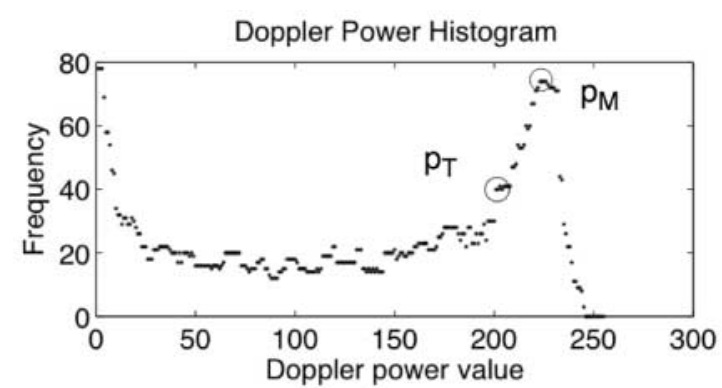

C

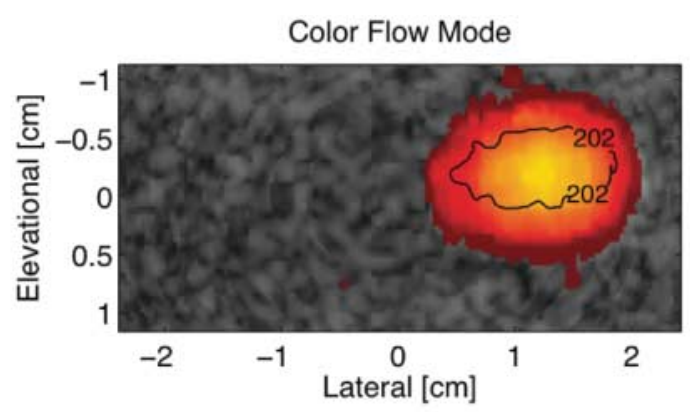

B

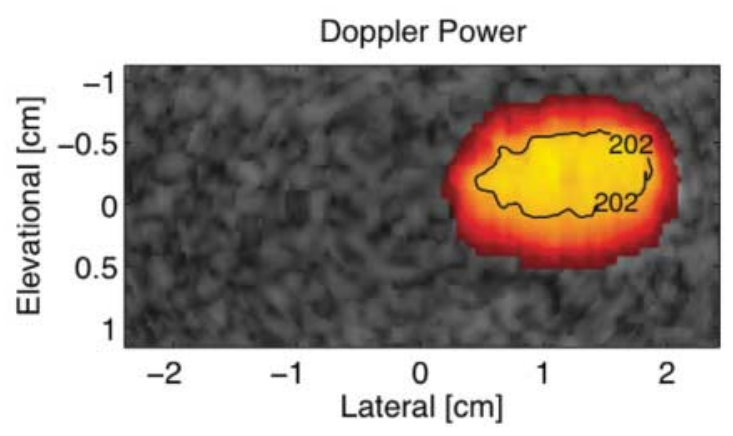

D

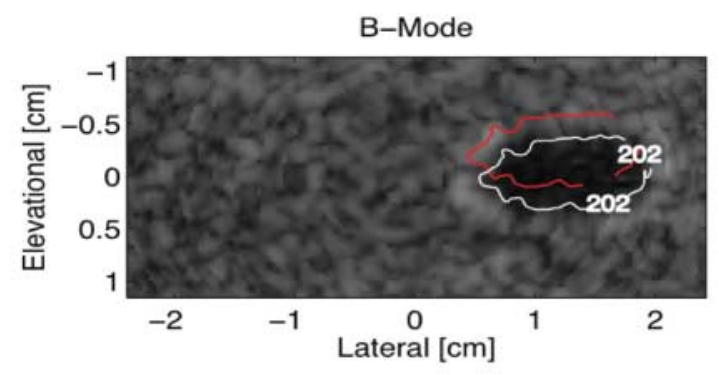


ing on their power value. Superpositioning of the $p_{T}$ contour and the B-mode data reveals both a slight misregistration of $\mathrm{CF}$ and B-mode in the elevational scan direction as well as the extent of partial volume effects (Figure 5D). Two contours are given: the red contour represents the original contour derived from the Doppler power; and the white contour is copied from the red contour and centered on the lumen to verify the choice of $p_{T}$ as $90 \%$ of $p_{M}$.

The Voluson system allows the user to set smoothing filter parameters, which reduce acquisition noise but also induce a spatial shift of the acquired Doppler voxels in the elevational direction because of the additional time of the acquisition. Filter parameters were held constant throughout the experiments for the presented data.

The B-mode in Figure 5, B-D, shows concentric rings, which are the result of scan conversion from the cartesian format exported by the scanner back to the original scan geometry format (raw data).

Volume flow was varied between 2 and $15 \mathrm{~mL} / \mathrm{s}$ $\left(110^{\circ}\right.$ Doppler angle) and computed by the power-weighted SIVV method. Figure 6 shows the resulting volume flow for 2 transducer orientations. Agreement between measurement and real flow was within $\approx 20 \%$, and all data were found to

Figure 6. Volume flow estimates for a range of flows between 2 and $15 \mathrm{~mL} / \mathrm{s}$. The $95 \%$ confidence intervals are shown as dashed lines and based on a linear regression, which is shown as a solid line. "O" reflects measurements with the transducer's aperture parallel to the tube axis, and " $X$ " reflects measurements with the transducer's aperture perpendicular to the tube axis.

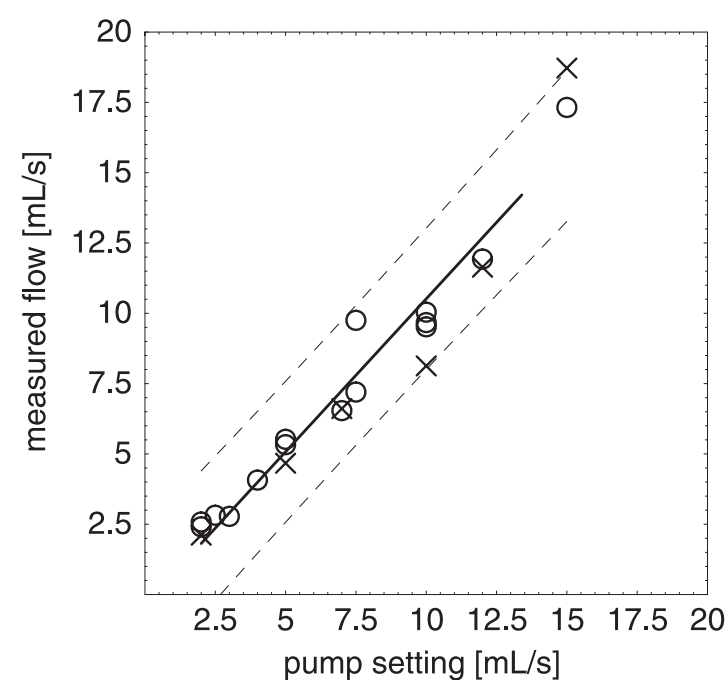

lie within the $95 \%$ confidence interval. The estimate for $15 \mathrm{~mL} / \mathrm{s}$ is high, which we possibly associate with the occurrence of debris/gas bubbles in the blood-mimicking fluid at high flow speeds $(>10 \mathrm{~mL} / \mathrm{s})$, which raised the Doppler power abnormally.

Doppler beam-to-flow angle variation was estimated for angles between $40^{\circ}$ and $140^{\circ}$, if $90^{\circ}$ corresponds to a purely laterally oriented flow tube (Figure 7). No data were collected for relative angles between $90^{\circ} \pm 20^{\circ}$ because they had low signal to noise due to the poor Doppler angle and the subsequent wall filter cutoff. In any case, one will always be able to steer $20^{\circ}$ to obtain a suitable Doppler angle. All data were within $\pm 15 \%$ of the true volume flow and also fell within the $95 \%$ confidence interval. Fit parameters for varying volume flow in Figure 6 were $Y=(1.09 \pm 0.06) \cdot X$ $-(0.36 \pm 0.52)$, and those for varying angles in Figure 7 were $Y=\left(5.9 \times 10^{-5} \pm 4.7 \times 10^{-4}\right) \cdot X-(1.02$ $\pm 0.05)$. The SE is given for each slope and intersept as the \pm component.

\section{Discussion}

Burns $^{2}$ stated that it is important that experimental studies of volume flow be presented together with a realistic assessment of the physical uncertainty of the measurement. We hope to

Figure 7. Normalized volume flow estimates for a range of relative Doppler angles. The flow pump was set to $5(0)$ and $10(+)$ $\mathrm{mL} / \mathrm{s}$, and most estimates are within $\pm 10 \%$ of the individual pump setting. All data lie within the $95 \%$ confidence interval shown as dashed lines. The dotted line represents a linear fit of the experimental values.

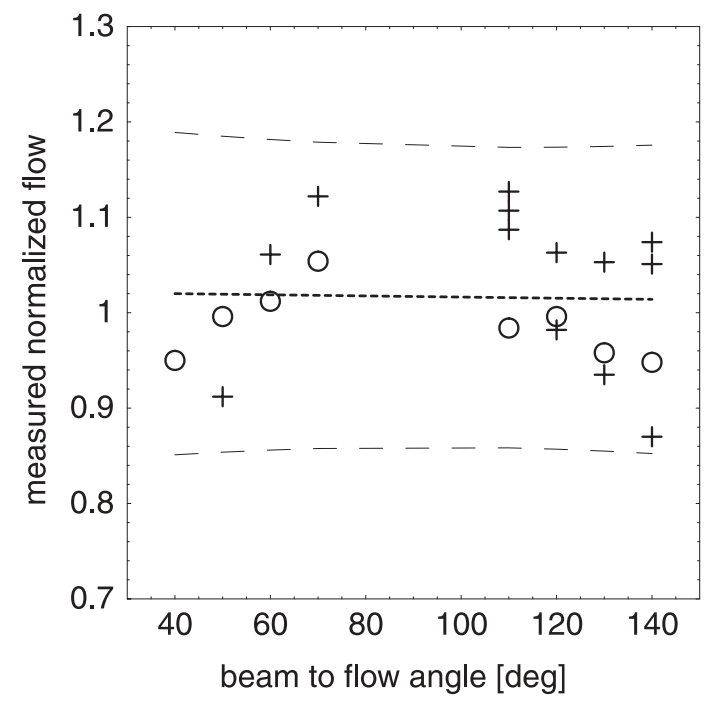

J Ultrasound Med 2006; 25:1305-1311 
have shown how this implementation of SIVV with automatic power weighting is an objective and therefore suitable technique to measure volume flow. In vitro experiments such as this one allow for comparisons with reference standards such as the pumping system used.

The selection of parameters for the powerweighted normalization method will require further investigation. Specifically, the selection of power values for the histogram that come from a range immediately above to below the integration plane may improve the technique. ${ }^{12}$ The number of voxels included will be a trade-off between reducing the effects of attenuation and having sufficient sampling for the histogram. Subsequent in vivo verification will need to be performed in a similar fashion to supply appropriate validation of the methods.

The velocities shown are in the lower range of physiologic values. Flow velocities in large arteries such as the aorta are as high as $50 \mathrm{~cm} / \mathrm{s}$, with flow reaching up to $100 \mathrm{~mL} / \mathrm{s}$. However, renal and hepatic flows are slower: $30 \mathrm{~cm} / \mathrm{s}(4 \mathrm{~mL} / \mathrm{s})$ and $64 \mathrm{~cm} / \mathrm{s}(30 \mathrm{~mL} / \mathrm{s})$, respectively. ${ }^{13-16}$ Moreover, there is no inherent reason for this technique not to work at higher physiologic flows. Our bench-top setup had limitations because of the occurrence of gas bubbles at higher flows, with detrimental effects on the Doppler processing.

In conclusion, power-weighted SIVV is a very promising technique for the acquisition of volume flow in vivo. The progressively greater availability of volumetric scanning using mechanical or electronic steering will make this easy to implement on clinical sonographic scanners. Medical diagnosis partially based on this physiologic parameter should become easy to achieve.

\section{References}

1. Bower S, Vyas S, Campbell S, Nicolaides KH. Color Doppler imaging of the uterine artery in pregnancy: normal ranges of impedance to blood flow, mean velocity and volume of flow. Ultrasound Obstet Gynecol 1992; 2:261-265.

2. Burns P. Measuring volume flow with Doppler ultrasound: an old nut. Ultrasound Obstet Gynecol 1992; 2:238-241.

3. Hottinger CF, Meindl JD. Blood flow measurement using the attenuation-compensated volume flowmeter. Ultrason Imaging 1979; 1:1-15.

4. Rubin JM. Flow quantification. Eur Radiol 1999; 9(suppl 3): S368-S371.
5. Embree PM, O'Brien WD Jr. The accurate ultrasonic measurement of the volume flow of blood by time domain correlation. Proc IEEE Ultrason Symp 1985; 2:963-966.

6. Rubin JM, Tuthill TA, Fowlkes JB. Volume flow measurement using Doppler and grey-scale decorrelation. Ultrasound Med Biol 2001; 27:101-109.

7. Sun $Y$, Ask P, Janerot-Sjoberg B, Eidenvall L, Loyd $D$, Wranne $B$. Estimation of volume flow rate by surface integration of velocity vectors from color Doppler images. J Am Soc Echocardiogr. 1995; 8:904-914.

8. Tsujino $H$, Shiki E, Hirama M, linuma K. Quantitative measurement of volume flow rate (cardiac output) by the multibeam Doppler method. J Am Soc Echocardiogr 1995; 8:621-630.

9. Berg S, Torp H, Haugen BO, Samstad S. Volumetric blood flow measurement with the use of dynamic 3-dimensional ultrasound color flow imaging. J Am Soc Echocardiogr 2000; 13:393-402.

10. Lui GY, Burns PN. The attenuation compensated C-mode flowmeter: a new Doppler method for blood volume flow measurement. Ultrason Symp 1997; 2:1285-1289.

11. Kripfgans $O D$, Rubin JM, Hall AL, Fowlkes JB. Vector Doppler imaging of a spinning disc ultrasound Doppler phantom. Ultrasound Med Biol 2006; 32:1037-1046.

12. Rubin JM, Adler RS, Fowlkes JB, et al. Fractional moving blood volume estimation using Doppler power imaging. Radiology 1995; 197:183-190.

13. Middleton WD, Kellman GM, Melson GL, Madrazo BL. Postbiopsy renal transplant arteriovenous fistulas: color Doppler US characteristics. Radiology 1989; 171:253-257.

14. Yamamoto T, Ogasawara Y, Kimura A, et al. Blood velocity profiles in the human renal artery by Doppler ultrasound and their relationship to atherosclerosis. Arterioscler Thromb Vasc Biol 1996; 16:172-177.

15. Hollerbach S, Schultze K, Muscholl M, Scholmerich J. Ultrasonography of the inferior vena cava (IVC) in the diagnosis and monitoring of therapy in patients with chronic congestive heart failure. Dtsch Med Wochenschr 2001; 126:129-133.

16. Henderson JM, Mackay GJ, Kutner M, Noe B. Volumetric and functional liver blood flow are both increased in the human transplanted liver. J Hepatol 1993; 17:204-207. 